\title{
Neonatal hormone changes and growth in lambs born to dams receiving differing nutritional intakes and selenium supplementation during gestation
}

\author{
Leticia E Camacho, Allison M Meyer, Tammi L Neville, Carolyn J Hammer, Dale A Redmer, \\ Lawrence P Reynolds, Joel S Caton and Kimberly A Vonnahme \\ Department of Animal Sciences, Center for Nutrition and Pregnancy, North Dakota State University, Fargo, \\ North Dakota 58108, USA \\ Correspondence should be addressed to K A Vonnahme; Email: kim.vonnahme@ndsu.edu
}

A M Meyer is now at Department of Animal Science, University of Wyoming, Laramie, Wyoming

\begin{abstract}
To investigate the effects of maternal selenium (Se) supplementation and nutritional intake during gestation on hormone changes, percentage body weight (BW) change, and organ mass in neonatal lambs, ewes were allocated to differing Se levels (adequate Se (ASe, $11.5 \mu \mathrm{g} / \mathrm{kg} \mathrm{BW)}$ or high Se (HSe, $77.0 \mu \mathrm{g} / \mathrm{kg} \mathrm{BW})$ ) initiated at breeding and nutritional intake (60\% (RES), $100 \%$ (CON), or $140 \%$ (HIGH) of NRC requirements) initiated at day 40 of gestation. At parturition, all lambs were removed from dams, fed common diets, and BW and blood samples were collected until day 19 . There was a Se $\times$ nutritional intake $\times$ day interaction for percentage BW change from birth. Lambs born to ASe-HIGH ewes tended to have decreased BW change compared with ASe-CON and ASe-RES groups on day 7. Lambs from HSe-HIGH ewes tended to have increased BW change compared with HSe-RES and HSe-CON groups from days 7 to 19. At birth, there was a Se $\times$ sex of offspring interaction, in which male lambs from HSe ewes had decreased cortisol concentrations compared with all other lambs. By 24 h, lambs from RES ewes had decreased cortisol compared with those from HIGH ewes, with lambs from CON ewes being intermediate. Lambs from RES- and CON-fed ewes had greater thyroxine than HIGH ewes at $24 \mathrm{~h}$. Organ masses on day 19 were mainly impacted by maternal nutritional intake and sex of the offspring. Birth weight alone did not predict growth performance during neonatal life. Moreover, despite a similar postnatal diet, maternal nutritional plane and Se status did impact neonatal endocrine profiles. Exact mechanisms of how neonatal endocrine status can influence later growth and development need to be determined.

Reproduction (2012) 144 23-35
\end{abstract}

\section{Introduction}

Maternal nutrition during pregnancy plays an important role in supporting adequate fetal and placental growth and development. Intrauterine growth restriction (IUGR) is associated with altered fetal organ development and subsequent performance of offspring (Wu et al. 2006, Neville et al. 2010, Reynolds et al. 2010, Vonnahme et al. 2010). Our laboratory has reported that maternal nutrient restriction during mid-to-late gestation in ewes decreased fetal body weight (BW; Reed et al. 2007) as well as birth weight (Neville et al. 2010). However, ewes given supranutritional selenium (Se) levels had greater fetal BW compared with ewes receiving adequate Se (ASe) levels (Reed et al. 2007).

IUGR is associated with accelerated growth and increased adiposity in the early neonatal period; although the mechanisms are unknown, they could include altered secretion or metabolism of thyroid hormones (DeBlasio et al. 2006). DeBlasio et al. (2006) suggested that placental restriction and small size at birth may increase activation of thyroxine $\left(\mathrm{T}_{4}\right)$ to triiodothyronine $\left(\mathrm{T}_{3}\right)$ and sensitivity of soft tissue to thyroid hormones, which may contribute to the accelerated neonatal growth following IUGR. Moreover, $\mathrm{T}_{4}$ is positively correlated with size at birth (DeBlasio et al. 2006). Additionally, Mellor \& Pearson (1977) demonstrated a positive correlation between lamb BW and concentrations of $\mathrm{T}_{4}$ at birth, which could be reflected by the effects of $T_{4}$ on fetal growth.

Glucocorticoids have major effects on the differentiation, morphology, and function of numerous tissues including the lungs, liver, kidneys, muscle, fat, and gastrointestinal tract (Challis et al. 2000, Fowden \& Forhead 2004). Glucocorticoids may also be essential for survival postnatally, as they induce maturation of several organs, including fetal lungs, brain, and gastrointestinal tract (Challis et al. 2000, Fowden \& Forhead 2004). 
However, the combined effects of dietary Se and nutritional intake on neonatal cortisol, $\mathrm{T}_{3}$, and $\mathrm{T}_{4}$ are not well defined. We hypothesized that supplementation with Se during gestation would offset the negative effects of limited fetal nutrient availability and therefore enhance growth and development of the offspring during neonatal life. Therefore, our objectives were to determine the effects of maternal dietary Se supplementation and plane of nutrition during gestation on neonatal growth, organ mass, and serum concentrations of cortisol, $\mathrm{T}_{3}$, and $\mathrm{T}_{4}$.

\section{Results}

The impacts of maternal diet on ewe growth throughout gestation, as well as gestation length, lamb BW at birth, size, average daily gain (ADG), and offspring adiposity has been previously reported by our laboratory (Meyer et al. 2010).

\section{Percentage weight change}

Our laboratory has previously published (Meyer et al. 2010) that in ASe ewes, lambs from RES ewes were lighter than $\mathrm{CON}$ and $\mathrm{HIGH}$; however, within the high Se (HSe) ewes, nutritional plane did not impact birth weight. Moreover, lambs from HSe-RES ewes were heavier than lambs from ASe-RES ewes (Meyer et al. 2010). Although male lambs were heavier $(P=0.02)$ at birth compared with female lambs $(4.73 \pm 0.14$ vs $4.31 \pm 0.10 \mathrm{~kg}$ ), there was no effect of sex of offspring, or its interaction with maternal Se supplementation, maternal nutritional intake, or day $(P \geq 0.35)$ on percentage weight change from birth to day 19 . However, there was a Se $\times$ nutritional intake $\times$ day interaction $(P=0.0002$; Fig. 1$)$. Percentage weight change from birth to days 1 and 3 was similar within each ewe dietary treatment $(P \geq 0.18)$. Thereafter, the percentage weight change from birth in all lambs increased until day $19(P \leq 0.02)$, with the exception of
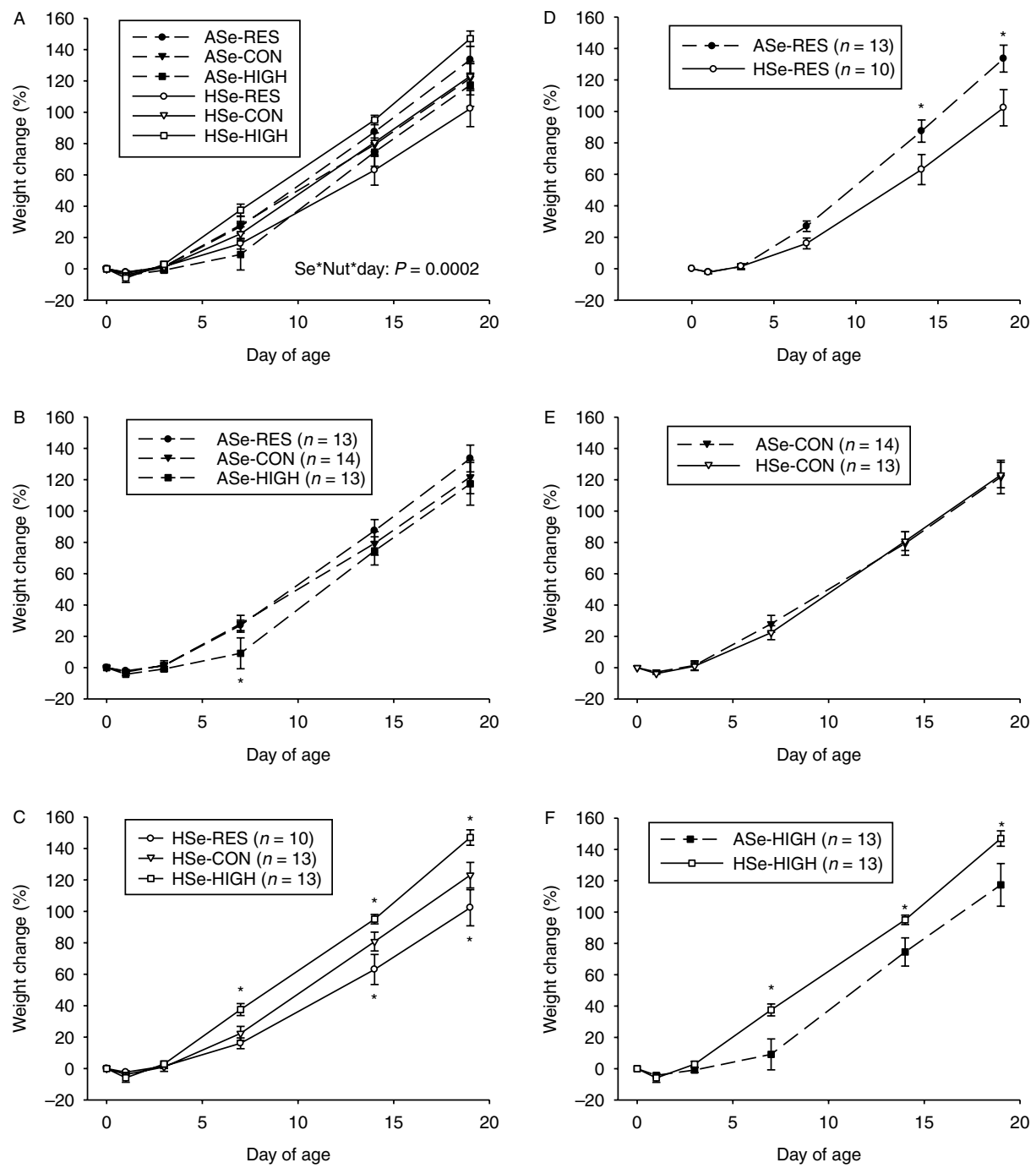

Figure 1 A maternal Se supplementation $\times$ maternal nutritional intake $\times$ day of age interaction (A) from birth to 19 days of age. Maternal Se supplementation treatments were $11.5 \mu \mathrm{g} \mathrm{Se} / \mathrm{kg}$ of body weight (adequate Se; ASe) or $77.0 \mu \mathrm{g}$ $\mathrm{Se} / \mathrm{kg}$ of body weight (high Se (HSe)) throughout gestation. Maternal nutritional intake treatments were RES $(60 \%$ of control), CON (control; $100 \%$ requirements for gestating ewe lambs), and $\mathrm{HIGH}(140 \%$ of control) fed from day 40 to term. Lambs were raised similarly after birth. Mean separations within a comparison are shown: (B) impacts of maternal nutritional plane within the ASe treatment; (C) impacts of maternal nutritional plane within the HSe treatment; (D) impacts of maternal Se status within the RES treatment; (E) impacts of Se status within the CON treatment; and $(\mathrm{F})$ impacts of Se status within the $\mathrm{HIGH}$ treatment. *Within a comparison, percentage change in lamb weight differed. 
ASe-HIGH, in which percentage weight change was similar $(P>0.16)$ from birth to day 7 and then increased $(P<0.0001)$ at days 14 and 19 . Lambs born to ASe-HIGH ewes had decreased $(P=0.03$; Fig. 1B) percentage weight change on day 7 compared with ASe-CON and ASe-RES groups, which did not differ $(P=0.98)$. In contrast, lambs from HSe-HIGH ewes had increased ( $P=0.01$; Fig. 1C) percentage weight change by day 7 compared with HSe-RES, which did not differ $(P=0.35)$ from HSe-CON. Moreover, lambs from ASe-HIGH ewes had a decrease $(P=0.001)$ in percentage weight change on day 7 compared with those from HSe-HIGH ewes (Fig. 1F). Percentage weight change by day 7 was similar between lambs from ASe-RES and HSe-RES $(P=0.16$; Fig. 1D) and ASe-CON and HSe-CON ewes $(P=0.62$; Fig. $1 \mathrm{E})$.

Although lambs from ASe ewes were not different among nutritional intake groups $(P \geq 0.13)$ by day 14 , lambs from HSe-CON and HSe-HIGH ewes had an increase $(P \leq 0.02)$ in percentage weight change compared with those from HSe-RES ewes (Fig. 1C). Furthermore, by day 14, lambs born to ASe-RES ewes had an increase $(P=0.003)$ in percentage weight change compared with those from HSe-RES (Fig. 1D), whereas lambs from ASe-CON were similar $(P=0.72)$ to those from HSe-CON (Fig. 1E), and lambs from ASe-HIGH ewes had a decrease $(P=0.01)$ in percentage weight change compared with those from HSe-HIGH (Fig. 1F).

By day 19, lambs from ASe-HIGH ewes tended to have a reduced $(P=0.06)$ percentage weight change compared with those from ASe-RES, with those from ASe-CON ewes being intermediate (Fig. 1B). Moreover, as maternal nutritional intake increased in lambs from HSe ewes, the percentage weight change in the lambs also increased ( $P \leq 0.01$; Fig. $1 C)$. Furthermore, by day 19, lambs born to ASe-RES ewes had an increase $(P=0.0004)$ in percentage weight change compared with those from HSe-RES ewes (Fig. 1D), whereas lambs from the ASe-CON group were similar $(P=0.74)$ to those from the HSe-CON group (Fig. 1E), and lambs from ASe$\mathrm{HIGH}$ ewes had a decrease $(P=0.0005)$ in percentage weight change compared with HSe-HIGH (Fig. 1F).

\section{Neonatal hormone profiles}

No maternal Se $\times$ maternal nutritional intake $\times$ offspring sex interactions were observed ( $P \geq 0.23$ ) for cortisol, $\mathrm{T}_{4}$, or $\mathrm{T}_{3}$ at birth or $24 \mathrm{~h}$ after birth. Moreover, there were no main effects $(P \geq 0.09)$ of maternal Se, maternal nutritional intake, or sex of offspring on $\mathrm{T}_{4}$ concentrations at birth, which averaged $115.1 \pm 3.6 \mathrm{ng} / \mathrm{ml}$. There were, however, main effects of maternal Se supplementation on cortisol concentrations ( $P=0.01$; Fig. $2 \mathrm{~A})$, maternal nutritional intake on $\mathrm{T}_{3}$ concentrations $(P=0.02$; Fig. 2B), and sex of offspring on $\mathrm{T}_{3}$ concentrations $(P=0.02$; Fig. 2C) at birth. Offspring from HSe ewes had decreased cortisol at birth $(P=0.01)$ compared with
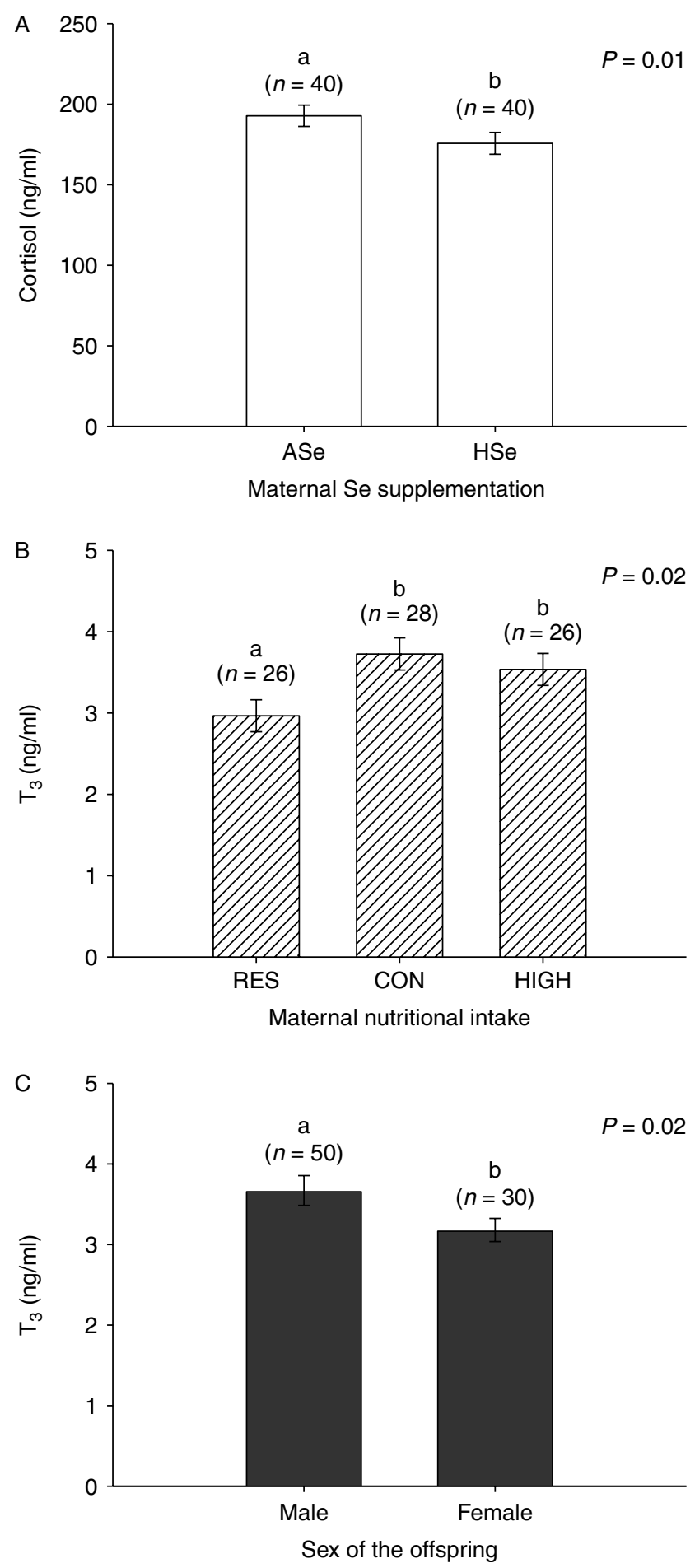

Figure 2 Circulating concentrations of cortisol $(A)$ and $T_{3}(B$ and $C)$ in blood collected immediately at birth in lambs born from ewes fed: $11.5 \mu \mathrm{g} \mathrm{Se} / \mathrm{kg}$ of body weight (adequate Se (ASe)) or $77.0 \mu \mathrm{g} \mathrm{Se} / \mathrm{kg}$ of body weight (high Se (HSe)) throughout gestation (A); maternal nutritional intake treatments were RES ( $60 \%$ of control), CON (control; $100 \%$ requirements for gestating ewe lambs), and $\mathrm{HIGH}(140 \%$ of control) fed from day 40 to term (B). Lamb sex influenced $T_{3}$ concentrations (C). ${ }^{a b}$ LSMean \pm s.E.M. within a panel differs; $P<0.05$. 
lambs born from ASe ewes (Fig. 2A). Ewes in the RES intake group gave birth to lambs with lower $(P \leq 0.05) \mathrm{T}_{3}$ concentrations compared with lambs from the CON- and $\mathrm{HIGH}$-fed ewes, which did not differ $(P=0.12$; Fig. 2B). Male lambs had increased $(P=0.02)$ $\mathrm{T}_{3}$ concentrations at birth compared with female lambs.

By $24 \mathrm{~h}$ after birth, maternal Se supplementation, maternal nutritional intake, and sex of the offspring did not $(P \geq 0.19)$ impact $\mathrm{T}_{3}$ concentrations, which averaged $3.99 \pm 0.13 \mathrm{ng} / \mathrm{ml}$. There was a main effect $(P \leq 0.04)$ of maternal nutritional intake on $\mathrm{T}_{4}$ and cortisol, with lambs from RES-fed ewes having increased $(P=0.04)$ circulating $\mathrm{T}_{4}$ but decreased $(P=0.01)$ cortisol concentrations compared with lambs from $\mathrm{HIGH}$-fed ewes (Fig. $3 \mathrm{~A}$ and $\mathrm{B}$ ). Moreover, $\mathrm{T}_{4}$ concentrations were also higher $(P=0.01)$ in lambs from $C O N-f e d$ ewes than lambs from $\mathrm{HIGH}$-fed ewes (Fig. 3A). Male lambs had less $(P=0.01)$ circulating cortisol concentrations than female offspring at $24 \mathrm{~h}$ after birth (Fig. 3C).

Concentrations of circulating $T_{3}, T_{4}$, and cortisol from days 3 to 19 were not affected $(P \geq 0.11)$ by maternal Se supplementation, maternal nutritional intake, offspring sex, or their interactions. There were offspring sex $\times$ day interactions for $\mathrm{T}_{3}(P=0.02$; Fig. $4 \mathrm{~A})$ and $\mathrm{T}_{4}$ concentrations $(P=0.005$; Fig. $4 \mathrm{~B})$ and a tendency for an offspring sex $\times$ day interaction for cortisol $(P=0.08$; Fig. 4C) concentration. Concentrations of $\mathrm{T}_{3}$ were higher $(P=0.02)$ on day 3 and decreased more from days 3 to 7 in male lambs compared with female lambs (Fig. 4A). Thereafter, concentrations of $\mathrm{T}_{3}$ were similar $(P>0.23)$ through day 19 in both sexes. Concentrations of $\mathrm{T}_{4}$ were similar $(P=0.61)$ on day 3 between male and female lambs, but male lambs had a tendency $(P=0.09)$ for a greater decline of $\mathrm{T}_{4}$ concentration by day 7 compared with female lambs (Fig. 4B). Thereafter, concentrations of $\mathrm{T}_{4}$ were similar $(P>0.17)$ between male and female lambs. Cortisol concentrations were increased $(P=0.03)$ in female compared with male lambs on day 3 . By day 7 , cortisol concentrations were similar $(P=0.76)$ and remained similar $(P>0.34)$ through the end of the study (Fig. 4C).

\section{Organ mass data}

We have previously reported (Meyer et al. 2010) that lamb BW on day 19 was less $(P \leq 0.01)$ in lambs from RES compared with lambs from $\mathrm{CON}$ and $\mathrm{HIGH}$ ewes (Table 1). Moreover, male lambs remained heavier $(P=0.03)$ than female lambs at day 19 (Table 2). There were no main effects of Se ( $P \geq 0.17$, data not shown) for any organ mass data collected; further, there were no maternal Se supplementation $\times$ maternal nutritional intake $\times$ offspring sex interactions $(P>0.15)$. Empty BW $($ EBW) was reduced $(P<0.01)$ in lambs from RES ewes compared with lambs from $\mathrm{CON}$ and $\mathrm{HIGH}$ ewes that did not differ $(P=0.83$; Table 1$)$. Moreover, male lambs had a greater $(P=0.03)$ EBW compared with female
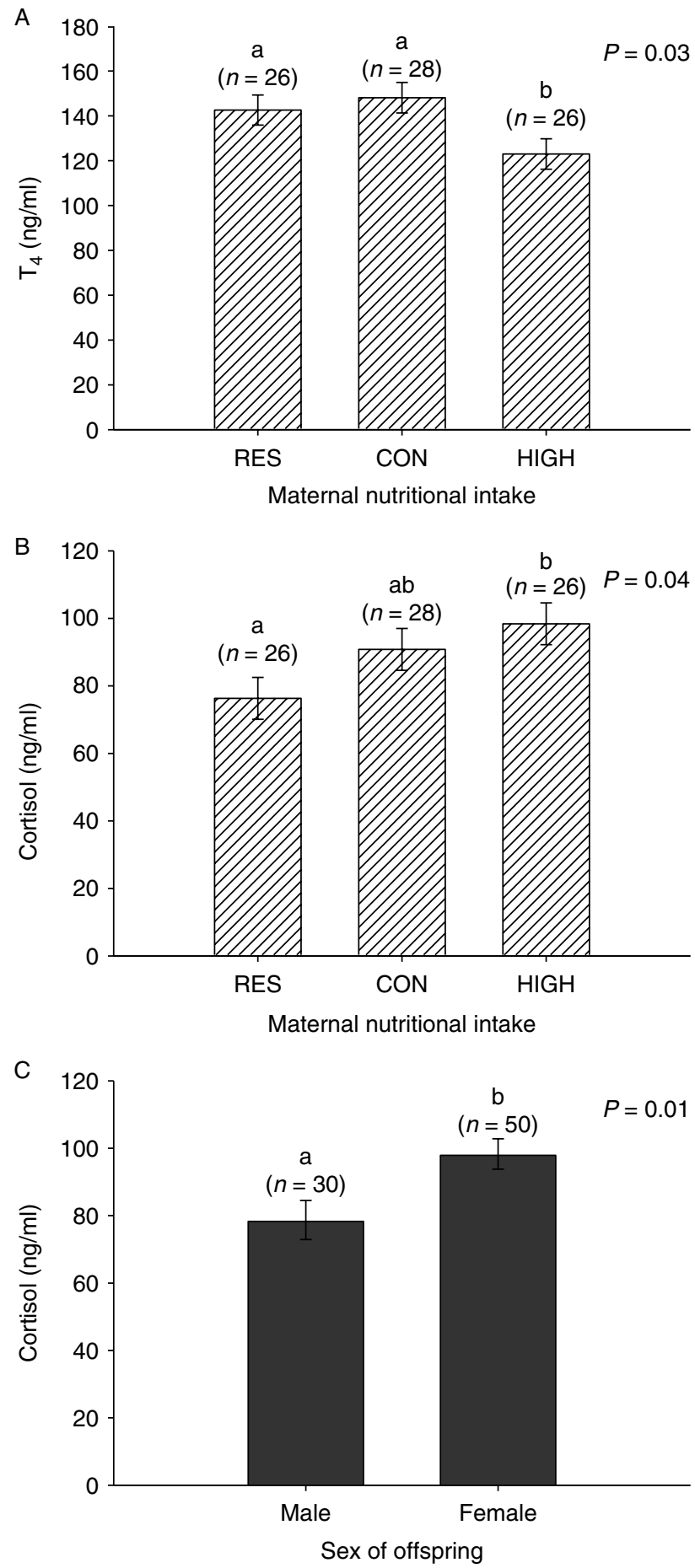

Figure 3 Circulating concentrations of $\mathrm{T}_{4}(\mathrm{~A})$ and cortisol $(\mathrm{B}$ and $\mathrm{C})$ in blood collected $24 \mathrm{~h}$ after birth in lambs born from ewes assigned to differing maternal nutritional intake treatments: RES (60\% of control), CON (control; $100 \%$ requirements for gestating ewe lambs), and HIGH (140\% of control) fed from day 40 to term (A and B). Lamb sex influenced cortisol concentrations (C). ${ }^{\mathrm{ab}} \mathrm{LSMean} \pm$ S.E.M. within a panel differs; $P<0.05$. 

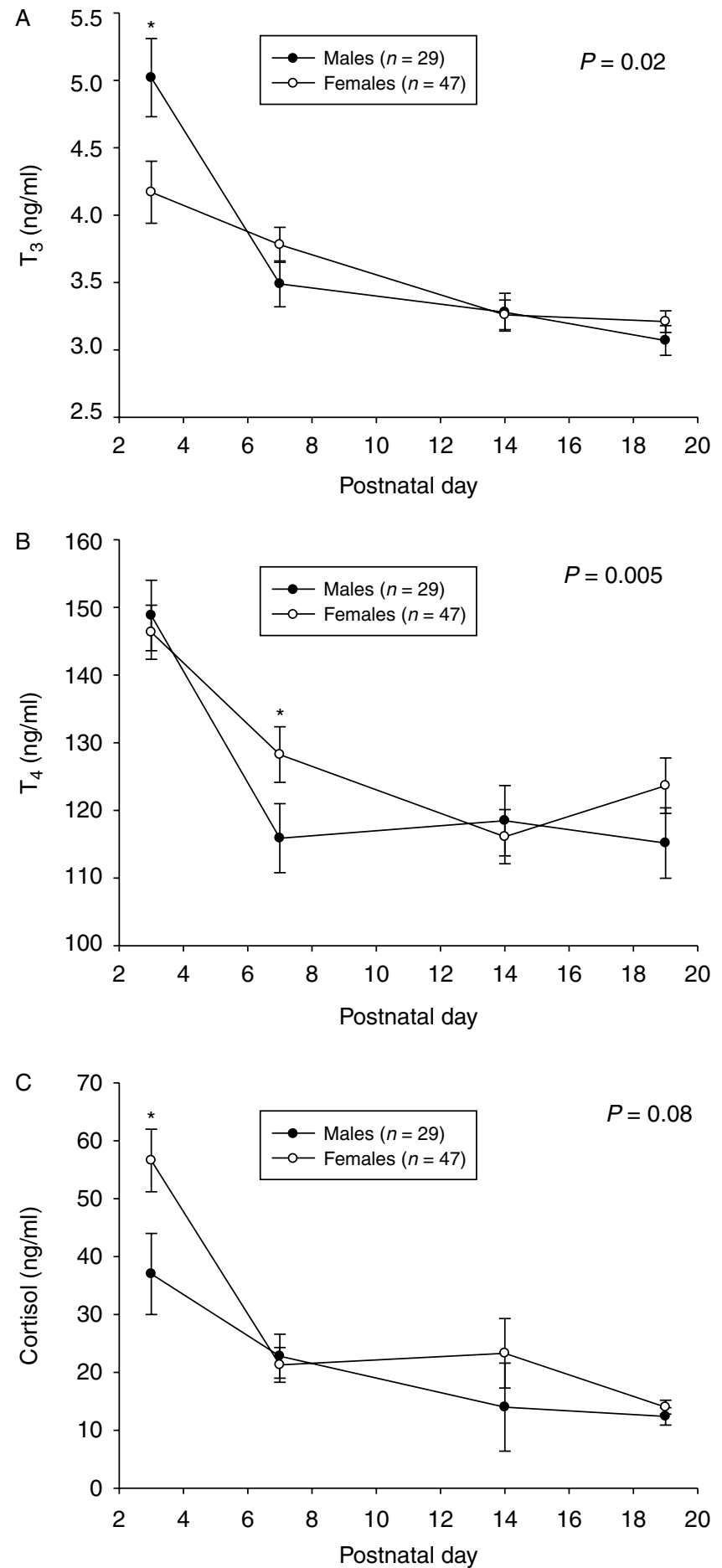

Figure 4 An offspring sex $\times$ day of age interaction for $T_{3}(A)$, cortisol $(B)$, and $\mathrm{T}_{4}(\mathrm{C})$ determined on days $3,7,14$, and 19 . ${ }^{*}$ Within a measure and day, concentrations differ; $P<0.05$.

lambs (Table 2). Whereas brain weight was not affected $(P=0.78)$ by maternal nutritional treatment, female lambs had decreased $(P=0.02)$ brain weight compared with male lambs. When brain weight was divided by EBW, lambs from RES ewes had a greater $(P<0.01)$ brain to EBW ratio compared with $\mathrm{CON}$ and $\mathrm{HIGH}$ ewes, which did not differ $(P=0.87)$. Male and female lambs did not differ $(P=0.36)$ in brain to EBW ratio. There was a main effect $(P \leq 0.03$; Table 1$)$ of maternal nutritional intake on blood weight (g and $\mathrm{g} / \mathrm{g}$ brain weight), where lambs born to ewes fed RES diets had less $(P \leq 0.03)$ blood mass than lambs from CON and HIGH-fed ewes. When expressed as blood weight to EBW ratio, there was a maternal Se $\times$ nutritional intake interaction $(P=0.03)$, with lambs from ASe-RES and ASe-HIGH ewes having a greater $(P<0.05)$ ratio compared with HSe-RES and HSe-HIGH respectively. Moreover, blood weight (g) was greater $(P=0.02$; Table 2$)$ and blood weight proportional to brain weight tended $(P=0.08)$ to be greater in male lambs compared with female lambs. There tended to be an interaction of maternal Se $\times$ nutritional intake on heart weight $(\mathrm{g})$, in which lambs from HSe-RES had lighter $(P \leq 0.005$; Table 3$)$ hearts than lambs from HSe$\mathrm{CON}$ and $\mathrm{HSe}-\mathrm{HIGH}$. When heart weight was expressed proportionally to brain weight, a main effect of maternal nutritional intake $(P<0.01)$ was observed, in which lambs from RES-fed ewes had lighter hearts compared with lambs from $\mathrm{CON}$ - and $\mathrm{HIGH}$-fed ewes. Females had lighter $(P=0.005$; Table 2$)$ hearts $(\mathrm{g})$ than males and tended $(P=0.10)$ to have decreased heart weight as a proportion of brain weight. Maternal nutrition did not affect ( $P \geq 0.20$; Table 1 ) neonatal lung mass (g and $\mathrm{g} / \mathrm{g}$ brain weight). There was a tendency $(P=0.08)$ for lambs from $\mathrm{HIGH}$ ewes to have a reduced $(P=0.03)$ lung to EBW ratio compared with lambs from RES ewes. However, male lambs had heavier $(P \leq 0.01$; Table 2$)$ lungs ( $g$ and $g / g$ brain weight) than female lambs.

Maternal nutritional intake affected $(P \leq 0.03$; Table 1$)$ total gastrointestinal, liver, and spleen masses ( $\mathrm{g}$ and $\mathrm{g} / \mathrm{g}$ brain weight), in that weights were less $(P \leq 0.04)$ for lambs born to RES ewes compared with CON- and $\mathrm{HIGH}$-fed ewes. There was a maternal Se $\times$ nutritional intake interaction $(P=0.02$; Table 3$)$ for liver to EBW ratio. Within the lambs from the ASe-fed ewes, RES had a greater $(P \leq 0.05)$ liver to EBW ratio compared with CON and $\mathrm{HIGH}$, which did not differ $(P=0.67)$. In lambs from HSe-fed ewes, CON had an increased $(P \leq 0.04)$ liver to EBW ratio compared with RES and $\mathrm{HIGH}$, which did not differ $(P=0.98)$. Lambs from ASe-RES ewes had an increased $(P=0.04)$ liver to EBW ratio compared with lambs from HSe-RES ewes, while lambs from ASe-CON ewes had a reduced $(P=0.05)$ liver to EBW ratio compared with lambs from HSe-CON (Table 3). There was a tendency $(P \leq 0.09$; Table 2$)$ for females to have a lighter gastrointestinal mass $(\mathrm{g})$ and spleen weight $(\mathrm{g} / \mathrm{g}$ brain weight) than males. Gastrointestinal mass as a proportion of brain weight was not affected by sex of offspring $(P=0.36)$. Liver (g and $g / g$ brain weight) and spleen weights $(\mathrm{g})$ were lighter $(P \leq 0.03$; Table 2$)$ in female lambs compared with male lambs. There was a maternal Se $\times$ offspring sex interaction $(P<0.01)$ for liver to EBW ratio (Table 4). While there was no difference 
Table 1 Impacts of maternal nutrition on organ mass weight (g, g/g brain weight, and g/g EBW) in 19 day-old lambs.

\begin{tabular}{|c|c|c|c|c|c|}
\hline \multirow[b]{2}{*}{ Item } & \multicolumn{3}{|c|}{ Nutrition treatments ${ }^{\mathrm{a}}$} & \multirow[b]{2}{*}{ S.E.M. } & \multirow[b]{2}{*}{$P$ value $^{\mathrm{b}}$} \\
\hline & $\operatorname{RES}(n=23)$ & $\mathrm{CON}(n=27)$ & $\mathrm{HIGH}(n=26)$ & & \\
\hline Live wt (kg) & $9.7^{*}$ & $11.3^{+}$ & $11.6^{+}$ & 0.4 & $<0.01$ \\
\hline EBW $(\mathrm{kg})$ & $8.7^{*}$ & $10.2^{+}$ & $10.3^{+}$ & 0.4 & $<0.01$ \\
\hline Brain (g) & 71.9 & 70.5 & 71.2 & 1.4 & 0.78 \\
\hline g/g EBW & $8.52 *$ & $7.19^{+}$ & $7.11^{+}$ & 0.34 & $<0.01$ \\
\hline Blood (g) & $538.9^{*}$ & $632.7^{+}$ & $640.8^{+}$ & 30.9 & 0.03 \\
\hline g/g Brain & $7.5^{*}$ & $9.0^{+}$ & $9.0^{+}$ & 0.4 & 0.01 \\
\hline g/g EBW ${ }^{\mathrm{A}}$ & 61.49 & 61.94 & 60.35 & 1.50 & - \\
\hline Heart $(g)^{A}$ & 62.0 & 71.8 & 70.4 & 2.4 & - \\
\hline g/g Brain & $0.87 *$ & $1.02^{+}$ & $0.99^{+}$ & 0.03 & $<0.01$ \\
\hline g/g EBW & 7.23 & 7.04 & 6.86 & 0.15 & 0.20 \\
\hline Lung (g) & 197.7 & 213.2 & 191.5 & 11.2 & 0.35 \\
\hline g/g Brain & 2.75 & 3.05 & 2.68 & 0.16 & 0.20 \\
\hline g/g EBW & $23.18^{*}$ & $21.56^{*,+}$ & $18.61^{+}$ & 1.51 & 0.08 \\
\hline Total gastrointestinal $^{\mathrm{C}}(\mathrm{g})$ & $498.6^{*}$ & $560.4^{+}$ & $572.2^{+}$ & 21.7 & 0.03 \\
\hline g/g Brain & $6.95^{*}$ & $8.02^{\dagger}$ & $8.04^{+}$ & 0.30 & 0.01 \\
\hline g/g EBW & 57.96 & 55.54 & 55.50 & 1.31 & 0.29 \\
\hline Liver $(\mathrm{g})$ & $272.6^{*}$ & $322.6^{+}$ & $313.7^{+}$ & 13.0 & 0.02 \\
\hline g/g Brain & $3.80^{*}$ & $4.61^{\dagger}$ & $4.40^{\dagger}$ & 0.19 & $<0.01$ \\
\hline g/g EBW ${ }^{A}$ & 31.46 & 31.64 & 29.93 & 0.70 & - \\
\hline Spleen (g) & $26.6^{*}$ & $33.4^{+}$ & $31.0^{+}$ & 1.4 & $<0.01$ \\
\hline g/g Brain & $0.37^{*}$ & $0.48^{+}$ & $0.44^{+}$ & 0.02 & $<0.01$ \\
\hline g/g EBW & 3.06 & 3.30 & 3.01 & 0.11 & 0.14 \\
\hline Pancreas ${ }^{\mathrm{A}}$ & 13.0 & 14.3 & 13.7 & 0.05 & - \\
\hline g/g Brain ${ }^{A}$ & 0.183 & 0.205 & 0.192 & 0.008 & - \\
\hline g/g EBW & 1.56 & 1.44 & 1.34 & 0.08 & 0.14 \\
\hline Kidney $(\mathrm{g})^{\mathrm{A}}$ & 59.8 & 64.1 & 69.5 & 2.8 & - \\
\hline g/g Brain ${ }^{A}$ & 0.83 & 0.91 & 0.98 & 0.04 & - \\
\hline g/g EBW ${ }^{A}$ & 6.89 & 6.31 & 6.66 & 0.15 & - \\
\hline Adrenal (g) & $1.04^{*}$ & $1.17^{+}$ & $1.10^{*,+}$ & 0.04 & 0.05 \\
\hline mg/g Brain ${ }^{A}$ & 14.7 & 16.7 & 15.5 & 0.05 & - \\
\hline g/g EBW & 0.12 & 0.12 & 0.11 & 0.005 & 0.15 \\
\hline Visceral adiposity $^{\mathrm{d}}(\mathrm{g})$ & $220.0^{*}$ & $283.3^{\dagger}$ & $310.4^{+}$ & 22.0 & 0.01 \\
\hline g/g Brain & $3.05^{*}$ & $4.04^{\dagger}$ & $4.38^{\dagger}$ & 0.30 & $<0.01$ \\
\hline g/g EBW ${ }^{\mathrm{A}}$ & 24.57 & 26.98 & 28.99 & 1.49 & - \\
\hline Thyroid (g) & 1.08 & 1.20 & 1.13 & 0.07 & 0.42 \\
\hline $\mathrm{mg} / \mathrm{g}$ Brain & 15.0 & 17.2 & 15.9 & 1.0 & 0.27 \\
\hline g/g EBW & 0.13 & 0.12 & 0.11 & 0.007 & 0.23 \\
\hline Testes (g) & 3.76 & 4.66 & 4.61 & 0.43 & 0.18 \\
\hline mg/g Brain & $50.4^{*}$ & $67.2^{+}$ & $62.3^{+}$ & 6.0 & 0.10 \\
\hline $\mathrm{mg} / \mathrm{g}$ EBW & 0.41 & 0.43 & 0.42 & 0.03 & 0.89 \\
\hline Ovaries (g) & 0.82 & 1.09 & 1.06 & 0.25 & 0.69 \\
\hline mg/g Brain & 11.5 & 16.0 & 14.9 & 3.7 & 0.62 \\
\hline mg/g EBW & 0.10 & 0.12 & 0.10 & 0.03 & 0.75 \\
\hline Uterus (g) & 2.56 & 2.98 & 2.90 & 0.19 & 0.24 \\
\hline mg/g Brain & 36.6 & 42.9 & 42.2 & 2.8 & 0.19 \\
\hline mg/g EBW & 0.31 & 0.32 & 0.30 & 0.02 & 0.51 \\
\hline
\end{tabular}

*, ${ }^{\dagger}$ LSMean \pm S.E.M. within a measure differs; $P<0.05$. EBW, empty body weight $=$ live weight minus digesta weight.

${ }^{a}$ Maternal nutritional treatments were RES (fed at $60 \%$ of CON), CON (control; $100 \%$ requirements for gestating ewe lambs), and $\mathrm{HIGH}(\mathrm{fed}$ at $140 \%$ of CON). ${ }^{\mathrm{b}}$ There was no effect of maternal Se supplementation $(P \geq 0.26)$ or maternal Se by maternal nutritional intake $(P \geq 0.11)$, maternal nutritional intake $\times$ offspring sex $(P>0.14)$, or maternal Se $\times$ maternal nutritional intake $\times$ offspring sex $(P>0.15)$ interactions for the measures presented in the table, except for ${ }^{A}$ blood wt (g/g EBW), heart wt (g), kidney wt (g, g/g brain, and g/g EBW), pancreas wt (g and g/g brain), liver wt (g/g EBW), visceral adiposity (g/g EBW), and adrenal wt (mg/g brain); see Tables 3, 4 and 5. ${ }^{\circ}$ Total gastrointestinal mass was calculated by summing the empty stomach, small and large intestinal masses. ${ }^{\mathrm{d}}$ Visceral adiposity was calculated by summing the omental, mesenteric, and perirenal fat masses.

$(P=0.61)$ between males and females in ASe-fed ewes, males from HSe ewes had a greater $(P<0.01)$ liver to EBW ratio compared with females from HSe ewes. Moreover, females from ASe ewes had a greater $(P=0.03)$ liver to EBW ratio compared with females from HSe ewes. Pancreatic mass (g and g/g brain) tended $(P \leq 0.09$; Table 3$)$ to be affected by maternal Se and was altered $(P \leq 0.03$; Table 5$)$ due to maternal nutritional intake and offspring sex. There also was a tendency for maternal Se $\times$ maternal nutritional intake interactions for pancreatic mass (g; Table 3$)$, which was less $(P=0.03)$ in $\mathrm{HSe}-\mathrm{HIGH}$ compared with HSe-CON and when expressed proportionally to brain weight was less in HSe-RES and HSe-HIGH lambs $(P \leq 0.04)$ compared with HSe-CON offspring. The maternal nutritional intake $\times$ offspring sex interaction (Table 5 ) resulted in females having less $(P \leq 0.003)$ pancreatic mass (g and g/g brain) compared with males within the CON-fed 
Table 2 Impacts of offspring sex on organ mass ( $g$, g/g brain weight, and $\mathrm{g} / \mathrm{g}$ EBW) in 19 day-old lambs.

\begin{tabular}{|c|c|c|c|c|}
\hline \multirow[b]{2}{*}{ Item } & \multicolumn{2}{|c|}{ Sex } & \multirow[b]{2}{*}{ S.E.M. } & \multirow[b]{2}{*}{$P$ value $^{\mathrm{a}}$} \\
\hline & $\begin{array}{l}\text { Female } \\
(n=47)\end{array}$ & $\begin{array}{c}\text { Male } \\
(n=29)\end{array}$ & & \\
\hline Live wt (kg) & 10.3 & 11.4 & 0.4 & 0.03 \\
\hline EBW $(\mathrm{kg})$ & 9.2 & 10.2 & 0.3 & 0.03 \\
\hline Brain wt (g) & 69.3 & 73.0 & 1.2 & 0.02 \\
\hline g/g EBW & 7.78 & 7.43 & 0.3 & 0.36 \\
\hline Blood wt (g) & 562.4 & 645.8 & 27.3 & 0.02 \\
\hline g/g Brain & 8.09 & 8.92 & 0.37 & 0.08 \\
\hline g/g EBW & 60.17 & 62.35 & 1.3 & 0.19 \\
\hline Heart wt (g) & 64.3 & 71.9 & 2.1 & $<0.01$ \\
\hline g/g Brain & 0.93 & 0.99 & 0.03 & 0.10 \\
\hline g/g EBW & 6.99 & 7.09 & 0.13 & 0.55 \\
\hline Lung wt (g) & 180.2 & 221.4 & 9.8 & $<0.01$ \\
\hline g/g Brain & 2.61 & 3.04 & 0.14 & 0.01 \\
\hline g/g EBW & 20.07 & 22.16 & 1.33 & 0.22 \\
\hline $\begin{array}{l}\text { Total gastrointestinal } \\
\text { wt (g) }\end{array}$ & 520.2 & 567.4 & 19.0 & 0.06 \\
\hline g/g Brain & 7.51 & 7.83 & 0.27 & 0.36 \\
\hline g/g EBW & 56.68 & 55.99 & 1.16 & 0.64 \\
\hline Liver (g) & 280.0 & 325.9 & 11.5 & $<0.01$ \\
\hline g/g Brain & 4.04 & 4.50 & 0.16 & 0.03 \\
\hline $\mathrm{g} / \mathrm{g} \mathrm{EBW}^{\mathrm{A}}$ & 30.24 & 31.78 & 0.61 & - \\
\hline Spleen wt (g) & 28.4 & 32.3 & 1.2 & 0.01 \\
\hline g/g Brain & 0.41 & 0.45 & 0.02 & 0.09 \\
\hline g/g EBW & 3.07 & 3.18 & 0.10 & 0.43 \\
\hline Pancreas ${ }^{\mathrm{A}}$ & 12.8 & 14.6 & 0.4 & - \\
\hline g/g Brain ${ }^{\mathrm{A}}$ & 0.185 & 0.202 & 0.007 & - \\
\hline g/g EBW & 1.43 & 1.46 & 0.07 & 0.72 \\
\hline Kidney & 61.0 & 67.9 & 2.4 & 0.03 \\
\hline g/g Brain & 0.88 & 0.94 & 0.03 & 0.17 \\
\hline g/g EBW & 6.59 & 6.64 & 0.13 & 0.76 \\
\hline Adrenal & 1.08 & 1.13 & 0.03 & 0.21 \\
\hline mg/g Brain ${ }^{\mathrm{A}}$ & 15.6 & 15.6 & 0.4 & - \\
\hline g/g EBW & 0.12 & 0.11 & 0.004 & 0.33 \\
\hline Visceral adiposity & 276.4 & 266.0 & 19.4 & 0.67 \\
\hline g/g Brain & 3.98 & 3.67 & 0.27 & 0.37 \\
\hline g/g EBW & 28.68 & 25.02 & 1.32 & 0.03 \\
\hline Thyroid & 1.12 & 1.15 & 0.06 & 0.76 \\
\hline mg/g Brain & 16.2 & 15.9 & 0.9 & 0.80 \\
\hline g/g EBW & 0.12 & 0.11 & 0.006 & 0.31 \\
\hline
\end{tabular}

EBW, empty body weight= live weight minus digesta weight

${ }^{a}$ There was no effect of maternal Se by offspring sex $(P \geq 0.17)$, maternal nutritional intake by offspring sex $(P>0.14)$, or maternal Se $\times$ maternal nutritional intake $\times$ offspring sex $(P>0.15)$ interactions for the measures presented in the table, except for ${ }^{A}$ pancreas wt ( $g$ and $g / g$ brain) and adrenal wt (mg/g brain), see Table 5; and liver (g/g EBW), see Table 4.

ewes. Pancreatic mass (g) within the HIGH-fed ewes was less $(P=0.01)$ in females than in males. Male offspring from RES-fed ewes had decreased $(P \leq 0.04)$ pancreatic mass (g) compared with those from $\mathrm{CON}$ - and $\mathrm{HIGH}$-fed ewes; however, when expressed as a proportion of brain weight, male offspring from RES-fed ewes were less than $(P=0.002)$ those from CON-fed ewes.

Maternal Se and maternal nutritional intake tended $(P=0.07$; Table 3$)$ to affect kidney weight $(\mathrm{g})$ and its proportion to brain weight $(P=0.05)$ and EBW $(P=0.03)$. Within HSe-fed ewes, kidney weight (g and $\mathrm{g} / \mathrm{g}$ brain weight) was least $(P \leq 0.03)$ in lambs from RES compared with those from $\mathrm{CON}$ - and $\mathrm{HIGH}$-fed ewes. When expressed as kidney/EBW, there was no impact
$(P>0.35)$ of maternal intake in HSe ewes; however, lambs from ASe-RES ewes had an increased $(P<0.02)$ ratio compared with $\mathrm{ASe}-\mathrm{CON}$ and $\mathrm{ASe}-\mathrm{HIGH}$, which did not differ $(P=0.14)$. Moreover, lambs from ASe-RES ewes had an increased $(P=0.05)$ kidney to EBW ratio compared with lambs from HSe-RES ewes. Kidney weight (g) was less ( $P=0.03$; Table 2$)$ in females compared with males; however, when expressed proportionally to brain weight, it did not differ between the sexes $(P=0.17)$. There was a main effect of maternal nutritional intake ( $P=0.05$; Table 1$)$ on absolute adrenal gland mass (g), in which adrenal glands from CON lambs were heavier $(P=0.02)$ than those from RES lambs, and those from $\mathrm{HIGH}$ lambs were intermediate. Adrenal gland mass (g) was not affected by offspring sex $(P=0.21$; Table 2). For adrenal gland weight expressed per unit of brain weight $(\mathrm{mg} / \mathrm{g})$, a tendency for a maternal nutritional intake $\times$ offspring sex interaction $(P=0.09$; Table 5) was detected. Although female adrenal gland mass (mg/g brain weight) did not differ $(P>0.29)$ due to maternal nutritional intake, male lambs from $C O N$ ewes had heavier adrenals $(P \leq 0.02)$ than did lambs from RES and HIGH ewes. Visceral adiposity (g and g/g brain weight) was less $(P \leq 0.04)$ in offspring from RES compared with offspring from $\mathrm{CON}$ - and $\mathrm{HIGH}$-fed ewes and was not altered $(P>0.36)$ due to offspring sex. When visceral adiposity was expressed per EBW, there tended to be a maternal Se $\times$ nutritional intake interaction $(P=0.08$; Table 3$)$. While maternal intake within ASe ewes did not influence $(P>0.15)$ lambs' $^{\prime}$ adiposity, within the HSe ewes, RES had less $(P<0.04)$ adiposity to EBW ratio compared with $\mathrm{CON}$ and $\mathrm{HIGH}$, which did not differ $(P=0.47)$. Furthermore, female lambs had a greater $(P=0.03)$ visceral adiposity to EBW ratio compared with male lambs (Table 2 ).

There was no effect $(P>0.23$; Tables 1 and 2$)$ of maternal nutritional intake or offspring sex on thyroid weight ( $\mathrm{g}$, mg/g brain weight and mg/g EBW). Maternal nutritional intake did not affect $(P=0.18$; Table 1$)$ testes weight $(\mathrm{g})$; however, there was a tendency $(P=0.10)$ for maternal nutritional intake to affect testes weight, with testes weight (g/g brain weight) of males from RES-fed ewes being lesser $(P \leq 0.10)$ than males from $\mathrm{CON}$ - and $\mathrm{HIGH}$-fed ewes. There were no effects $(P>0.18$; Table 1$)$ of maternal nutrition on ovarian weight $(\mathrm{g}, \mathrm{mg} / \mathrm{g}$ brain weight and $\mathrm{mg} / \mathrm{g}$ EBW) or uterine weight ( $\mathrm{g}$, $\mathrm{mg} / \mathrm{g}$ brain weight and $\mathrm{mg} / \mathrm{g}$ EBW).

\section{Discussion}

Previous work from our laboratory has demonstrated that maternal Se supplementation much above the requirements may help to offset some of the negative impacts of maternal dietary restriction, which in our model leads to IUGR (Reed et al. 2007, Meyer et al. 2010). In this study, our focus was how neonatal growth, $T_{3}, T_{4}$, and cortisol concentrations would be impacted by maternal diet, as 
Table 3 Impacts of maternal Se supplementation ${ }^{a}$ and maternal nutritional intake on organ mass weight (g, g/g brain weight and g/g EBW) in 19 dayold lambs.

\begin{tabular}{|c|c|c|c|c|c|c|c|c|}
\hline \multirow[b]{2}{*}{ Item } & \multicolumn{3}{|c|}{ ASe } & \multicolumn{3}{|c|}{ HSe } & \multirow[b]{2}{*}{ S.E.M. } & \multirow{2}{*}{$\begin{array}{c}\text { Se } \times \text { Nut } \\
P \text { value }\end{array}$} \\
\hline & $\operatorname{RES}(n=13)$ & $\mathrm{CON}(n=14)$ & $\mathrm{HIGH}(n=13)$ & RES $(n=10)$ & $\operatorname{CON}(n=13)$ & $\mathrm{HIGH}(n=13)$ & & \\
\hline Blood wt (g) & 583.4 & 597.0 & 654.7 & 494.4 & 668.3 & 626.9 & 45.7 & 0.15 \\
\hline g/g EBW & $64.49^{*}$ & $59.77^{*,+}$ & $61.78^{*}$ & $58.50^{\dagger}$ & $64.11^{*,+}$ & $58.93^{\dagger}$ & 2.27 & 0.03 \\
\hline Heart wt (g) & $65.0^{*,+}$ & $68.0^{*,+, \neq}$ & $68.4^{+, \neq}$ & $59.1^{*}$ & $75.5^{\ddagger}$ & $72.5^{+, \neq}$ & 3.5 & 0.10 \\
\hline g/g Brain & 0.90 & 0.97 & 0.95 & 0.84 & 1.08 & 1.04 & 0.05 & 0.11 \\
\hline Pancreas & $12.9^{*}$ & $13.5^{*,+}$ & $14.5^{*, \dagger}$ & $13.2^{*,+}$ & $15.1^{\dagger}$ & $12.9^{*}$ & 0.7 & 0.06 \\
\hline g/g Brain & $0.179 *$ & $0.192^{*,+}$ & $0.201^{*,+}$ & $0.187^{*}$ & $0.219^{\dagger}$ & $0.184^{*}$ & 0.011 & 0.09 \\
\hline Liver (g) & 292.6 & 305.8 & 309.4 & 252.5 & 339.5 & 318.0 & 19.3 & 0.11 \\
\hline g/g EBW & $32.89 *$ & $30.37^{+}$ & $29.81^{\dagger}$ & $30.03^{+}$ & $32.91^{*}$ & $30.05^{\dagger}$ & 1.01 & 0.02 \\
\hline $\begin{array}{l}\text { Visceral adi- } \\
\text { posity (g) }\end{array}$ & 244.6 & 263.4 & 290.1 & 195.4 & 303.2 & 330.7 & 32.4 & 0.22 \\
\hline $\mathrm{g} / \mathrm{g}$ EBW & $26.64^{*,+}$ & $25.08^{*,+}$ & $27.06^{*,+}$ & $22.49^{*}$ & $28.88^{+, \neq}$ & $30.92^{\ddagger}$ & 2.19 & 0.08 \\
\hline Kidney (g) & $64.1^{*,+, \neq}$ & $60.3^{*, \neq}$ & $67.0^{+, \neq}$ & $55.5^{*}$ & $67.9^{+, \neq}$ & $72.0^{+}$ & 4.1 & 0.07 \\
\hline g/g Brain & $0.88^{*,+}$ & $0.86^{*,+}$ & $0.92^{+, \neq}$ & $0.78^{*}$ & $0.97^{+, \neq}$ & $1.03^{\ddagger}$ & 0.05 & 0.05 \\
\hline g/g EBW & $7.16^{*}$ & $6.08^{+}$ & $6.51^{t, \neq}$ & $6.61^{\ddagger}$ & $6.55^{+, \neq}$ & $6.81^{*, \neq}$ & 0.21 & 0.03 \\
\hline
\end{tabular}

${ }^{*},{ }^{,+} \neq$LSMean \pm S.E.M. within a measure differs; $P<0.05$. EBW, empty body weight = live weight minus digesta weight.

${ }^{a}$ Maternal Se supplementation treatments were $11.5 \mu \mathrm{g}$ Se/kg of body weight (adequate Se; ASe) or $77.0 \mu \mathrm{g} \mathrm{Se} / \mathrm{kg}$ of body weight (high Se (HSe)) throughout gestation. Maternal nutritional intake treatments were RES (60\% of control), CON (control; $100 \%$ requirements for gestating ewe lambs), and $\mathrm{HIGH}(140 \%$ of control) fed from day 40 to term. There was no effect of maternal Se supplementation $(P \geq 0.24)$ and no maternal Se $\times$ maternal nutritional intake $\times$ offspring sex interactions were significant $(P>0.15)$ for blood, heart, kidney, and pancreas measurements. There was a maternal Se supplementation $\times$ offspring sex interaction for liver (g/g EBW) as depicted in Table 4.

well as how neonatal organ mass may have been influenced by their uterine environment. In the current study, lambs were removed immediately from their dams in order to more closely assess the impacts of the maternal environment during gestation. We have previously reported that maternal diet (both nutritional intake and Se supplementation) can impact colostrum/ milk components as well as colostrum/milk volumes (Swanson et al. 2008, Meyer et al. 2011). Maternal Se supplementation throughout gestation did impact the growth patterns of offspring born from dams fed differing levels of nutrition from mid-to-late gestation. Two interesting responses observed on percentage weight change were the decreased weight change in lambs born to ASe-HIGH-fed ewes and the increased percentage weight change in lambs born to $\mathrm{HSe}-\mathrm{HIGH}$-fed ewes. Fry et al. (2010) demonstrated that jejunal cell proliferation was greater in lambs born to ASe-HIGH-fed ewes than $\mathrm{HSe}-\mathrm{HIGH}$-fed ewes. However, no differences in jejunal morphology suggested increased cell turnover and thus increased nutrient usage by ASe-HIGH. This may have diverted nutrients from whole-body growth contributing to decreased percentage weight change in lambs from this treatment.

Se supplementation did not appear to impact the growth of offspring in this study if the dams were fed adequately throughout gestation. It is interesting to think that supranutritional Se may be protective when nutritional intake is not ideal (Meyer et al. 2010). When ewes were supplemented with HSe, there was also a reduction in lamb cortisol concentrations at birth. Within $24 \mathrm{~h}$, this effect ceased and only nutritional intake impacted cortisol concentrations, with increased offspring cortisol as maternal intake increased. Dietary Se supplementation has been shown to enhance humoral immune function and increase IgG absorption (Knight \& Tyznik 1990, Kamada et al. 2007). Our laboratory has suggested that maternal Se supplementation and nutritional intake level during pregnancy can affect neonatal health and its ability to obtain passive immunity via lgG (Hammer et al. 2011). In a study similar to the current experiment (note, this study used a

Table 4 Impacts of maternal selenium ${ }^{a}$ and offspring sex on organ mass weight (g and g/g EBW) in 19 day-old lambs.

\begin{tabular}{|c|c|c|c|c|c|c|}
\hline \multirow[b]{2}{*}{ Item } & \multicolumn{2}{|c|}{ ASe } & \multicolumn{2}{|c|}{ HSe } & \multirow[b]{2}{*}{ S.E.M. } & \multirow[b]{2}{*}{$P$ value } \\
\hline & Female $(n=22)$ & Male $(n=17)$ & Female $(n=24)$ & Male $(n=12)$ & & \\
\hline $\begin{array}{l}\text { Liver (g) } \\
\text { g/g EBW }\end{array}$ & $\begin{array}{l}280.2 \\
31.30^{*}\end{array}$ & $\begin{array}{l}324.9 \\
30.75^{*,+}\end{array}$ & $\begin{array}{l}279.8 \\
29.18^{+}\end{array}$ & $\begin{array}{l}326.9 \\
32.81 *\end{array}$ & $\begin{array}{c}17.4 \\
0.92\end{array}$ & $\begin{array}{r}0.93 \\
<0.01\end{array}$ \\
\hline
\end{tabular}

${ }^{*,+}$ LSMean \pm S.E.M. within a measure differs; $P<0.05$. EBW, empty body weight $=$ live weight minus digesta weight.

${ }^{a}$ Maternal Se supplementation treatments were $11.5 \mu \mathrm{g}$ Se/kg of body weight (adequate Se; ASe) or $77.0 \mu \mathrm{g}$ Se/kg body weight (high Se; HSe) throughout gestation. For liver (g/g EBW), there was a main effect of sex (see Table 2), and an effect of maternal Se $\times$ maternal nutritional intake was significant $(P=0.02)$ and is presented in Table 3. 
Table 5 Impacts of maternal nutritional intake and offspring sex on organ mass weight (g and g/g brain weight) in 19 day-old lambs .

\begin{tabular}{|c|c|c|c|c|c|c|c|c|}
\hline \multirow[b]{2}{*}{ Item } & \multicolumn{2}{|c|}{ RES } & \multicolumn{2}{|c|}{ CON } & \multicolumn{2}{|c|}{ HIGH } & \multirow[b]{2}{*}{ S.E.M. } & \multirow{2}{*}{$\begin{array}{c}\text { Nut } \times \text { Sex } \\
P \text { value }\end{array}$} \\
\hline & $\begin{array}{l}\text { Female } \\
(n=12)\end{array}$ & $\begin{array}{c}\text { Male } \\
(n=11)\end{array}$ & $\begin{array}{l}\text { Female } \\
(n=20)\end{array}$ & $\begin{array}{l}\text { Male } \\
(n=7)\end{array}$ & $\begin{array}{l}\text { Female } \\
(n=15)\end{array}$ & $\begin{array}{c}\text { Male } \\
(n=11)\end{array}$ & & \\
\hline Pancreas (g) & $13.2^{*,+}$ & $12.9^{*}$ & $12.7^{*}$ & $16.0^{\ddagger}$ & $12.5^{*}$ & $14.9^{+, \neq}$ & 0.9 & 0.03 \\
\hline g/g Brain & $0.191^{*}$ & $0.175^{*}$ & $0.182^{*}$ & $0.228^{+}$ & $0.182 *$ & $0.203^{*,+}$ & 0.011 & 0.01 \\
\hline Adrenal wt (g) & 1.04 & 1.05 & 1.11 & 1.24 & 1.10 & 1.10 & 0.06 & 0.41 \\
\hline $\mathrm{mg} / \mathrm{g}$ Brain & $15.0^{*}$ & $14.3^{*}$ & $15.8^{*,+}$ & $17.6^{+}$ & $15.9^{*,+}$ & $15.0^{*}$ & 0.9 & 0.09 \\
\hline
\end{tabular}

${ }^{*, t, \neq}$ LSMean \pm S.E.M. within a measure differs; $P<0.05$.

${ }^{\mathrm{a}}$ Maternal nutritional intake treatments were RES ( $60 \%$ of control), CON (control; $100 \%$ requirements for gestating ewe lambs), and $\mathrm{HIGH}(140 \%$ of control) fed from day 40 to term. There was no effect of maternal Se $\times$ maternal nutritional intake $\times$ offspring sex interactions $(P>0.15)$ for measures presented in the table.

different Se source), maternal Se and nutritional intake impacted lamb serum IgG concentrations at $24 \mathrm{~h}$ after birth (Hammer et al. 2011; Exp. 1). In animals from the current study (Hammer et al. 2011, Exp. 2), lambs from RES ewes had greater serum IgG than $\mathrm{CON}$ or $\mathrm{HIGH}$, suggesting increased $\lg \mathrm{G}$ uptake postnatally. Moreover, female lambs had increased times of medical treatment (i.e. indicator of morbidity; Hammer et al. 2011). By $24 \mathrm{~h}$ postpartum, we observed (this study) that cortisol concentrations are elevated in females and in lambs from $\mathrm{HIGH}$-fed ewes. While the exact role of stress or cortisol on gut closure and IgG uptake in ruminants is still questionable (Stott 1980), we find our associations between cortisol and IgG patterns of interest. The capacity to take up immunoglobulins may associate with factors such as fetal age and plasma cortisol levels (Johnston \& Stewart 1986). Sangild etal. (1999) observed no significant correlation between cortisol and IgG $15 \mathrm{~h}$ after birth in premature or mature calves. Changes in morphology and function of the tissues have been associated with glucocorticoids, which activate many biochemical processes. Changes in tissue function may have little or no function in utero but could be essential for neonatal survival (reviewed by Fowden \& Forhead (2004)).

Maternal dietary Se supplementation had no effect on neonatal serum $T_{3}$ and $T_{4}$ concentrations. However, lambs born to RES-fed ewes had decreased $\mathrm{T}_{3}$ concentrations compared with those born to $\mathrm{CON}$ - and $\mathrm{HIGH}$ fed ewes. In addition, male lambs had more $T_{3}$ than female lambs. Conversely, $\mathrm{T}_{4}$ was greater in lambs born to RES- and CON-fed ewes compared with those born to $\mathrm{HIGH}$-fed ewes. Thyroid hormone concentrations from fetuses are reduced during hypoxemic conditions associated with IUGR (Fowden \& Forhead 2004). DeBlasio et al. (2006) suggested that placental restriction and small size at birth may increase activation of $T_{4}$ to $T_{3}$ and sensitivity of soft tissue to thyroid hormones, which may contribute to the accelerated growth following IUGR. Moreover, Mellor \& Pearson (1977) demonstrated a positive correlation between lamb BW and concentrations of $\mathrm{T}_{4}$ at birth, which could be related to effects of $\mathrm{T}_{4}$ on fetal growth. Both $\mathrm{T}_{3}$ and $\mathrm{T}_{4}$ seem to influence synthesis and secretion of $\mathrm{GH}$ as well as insulin-like growth factor 1 (IGF1) and IGF binding proteins. Schneider et al. (2002) suggested that conversion of inactive $T_{4}$ to active $T_{3}$ by hepatic deiodinase is controlled by $\mathrm{GH}$. An interesting finding in the current study was that maternal Se supplementation did not affect $T_{3}$ and $T_{4}$ concentrations even though Se is being known to participate in the conversion of $T_{4}$ to $T_{3}$ (Beckett et al. 1987) through selenoprotein type 1 iodothyronine 5-deiodinase (Arthur et al. 1990). The lack of Se effect may be that dams were offered adequate and HSe intake rather than a restricted Se intake, which is where selenoproteins seem to differ (i.e. during inadequate Se intake). The fetal thyroid gland in humans becomes functional after mid gestation; therefore, thyroid hormone transfer from the dam to the conceptus through the placenta is very important (Contempre et al. (1993) as cited by Poppe \& Ginoer (2003)). Development of the fetal central nervous system (CNS) during the second third of pregnancy largely depends on maternal thyroid hormones. In this study, lambs born to RES-fed ewes had lighter brain weight compared with lambs born to $\mathrm{CON}$ - and $\mathrm{HIGH}$-fed ewes, while there was no difference in thyroid gland weight of offspring.

In the current study, lambs born to RES-fed ewes had lighter live weight, as well as several organ masses, compared with lambs born to $\mathrm{CON}$ - and $\mathrm{HIGH}$-fed ewes at 19 day. In several cases, when organ masses are expressed per EBW, these relative organ masses are not different from the $\mathrm{CON}$-fed animals. However, because live BW and EBW are influenced by maternal diet, we based organ mass on an organ that was not impacted by maternal diet, the brain. The majority of the organs that were influenced by maternal dietary intake were also influenced when organ mass was expressed per brain weight. Other researchers have used the brain as an indicator to determine asymmetric vs symmetric growth restriction, as the brain is spared during the time of maternal nutrient restriction used in this study (Anthony et al. 2003). Symmetric IUGR is often associated with genetic abnormalities or infections that may occur early in pregnancy (Anthony et al. 2003). Asymmetric IUGR is more common and often associated with placental insufficiency (Anthony et al. 2003), potentially due to 
redistribution of blood flow to the fetus, allowing for brain sparing (Newton et al. 1987, Morrison 2008). Asymmetric IUGR has been documented in several mammalian species including sheep (Jensen et al. 1999, Anthony et al. 2003, Lang et al. 2003), swine (Widdowson 1971, Bauer et al. 1998), guinea pigs (Saintonge \& Rosso 1981), and humans (Pearce \& Campbell 1985). As we believe that our nutritional restriction model is due to differences in placental function, independent of size (Lekatz et al. 2010, 2011), basing offspring organ mass data per unit brain (i.e. a denominator that is consistent regardless of maternal nutritional intake) may be advantageous. While our model of overnutrition (from mid-to-late pregnancy) appears not to impact neonatal organ weights, a model of overnutrition from conception through parturition (Wallace et al. 2000) resulted in decreased brain, lung, heart, liver, kidneys, pancreas, spleen, and thyroid weights. While our study did use young, primiparous ewe lambs, they may have been slightly different in age (i.e. number of estrous cycle before conception) than those used in Wallace et al.'s (2000) study. We hypothesize that the differences between this model (Wallace et al. 2000) of overnutrition and the one in the current study is the level and duration of the overnutrition. Data from this study show only limited impacts of maternal Se on organ mass at 21 day. The alterations in organ masses in the current study were due to maternal diet, or sex of offspring, and could be linked to offspring cortisol concentrations. Glucocorticoids can impact the differentiation of several tissues including the lungs, liver, kidneys, muscle, fat, and gastrointestinal tract (reviewed by Fowden \& Forhead (2004)).

In conclusion, despite similar postnatal diets, maternal Se and nutritional intake during gestation can impact offspring percentage weight change during the first 19 days of life. Organ mass by 19 days of life appears to be more influenced by maternal nutritional intake and sex of the offspring. However, it appears that maternal diet has a greater impact on cortisol and thyroid hormone concentrations for the first $24 \mathrm{~h}$. The first hours postnatal are critical for immunoglobulin absorption, and cortisol and thyroid hormone concentrations may be essential for proper neonatal survival. It is still unclear how altered thyroid hormone and cortisol levels in the first $24 \mathrm{~h}$ impact the differential growth patterns that were observed in offspring from this study. Moreover, future studies are needed to determine whether these maternal diet-induced changes in hormones can impact physiological functions of the offspring.

\section{Materials and Methods}

Institutional Animal Care and Use Committees at North Dakota State University (NDSU), Fargo, and the USDA, ARS, U.S.
Sheep Experiment Station (USSES; Dubois, ID, USA) approved the animal care procedures used for this study.

\section{Animal management and diets}

\section{Gestation}

At the USSES, 178 Rambouillet ewe lambs (initial age $=240$ \pm 17 day; initial $\mathrm{BW}=52.1 \pm 6.2 \mathrm{~kg}$ ) were synchronized for estrus and divided into six groups for breeding $(n=29$ or 30/group). Ewe lambs were synchronized using intravaginal sponges (60 mg medroxyprogesterone acetate; Veramix, Pharmacia \& Upjohn, Orangeville, ON, Canada) and PGF2 $\alpha$ (15 mg; Lutalyse, Pharmacia \& Upjohn, Kalamazoo, MI, USA) over a 10 days period. More specifically, on day 0 , sponges were inserted; on day 7, two injections of PGF2 $\alpha$ (7.5 mg/injection) were administered $\sim 4 \mathrm{~h}$ apart; on day 10 , sponges were removed. On days 11-16, ewes were exposed to Rambouillet rams ( $n=12$, two/pen). Marking paint was placed on the rams' briskets to facilitate identification of ewes that the rams had attempted to breed. 'Marked' ewes were then stratified by BW and assigned randomly to one of two treatment pens. After rams were removed from the breeding pens, each pen was randomly assigned to Se treatments, which were ASe (3.5 $\mu \mathrm{g} \mathrm{Se/kg} \mathrm{BW} \mathrm{per} \mathrm{day)} \mathrm{or} \mathrm{HSe} \mathrm{(65} \mathrm{\mu g} \mathrm{Se/kg} \mathrm{BW} \mathrm{per} \mathrm{day).}$ Se was provided to HSe ewes in the form of Se-enriched wheat mill run, which resulted from on-site processing of wheat grown in a seleniferous region near Pierre, SD, USA, whereas the sole source of Se for ewes on the ASe treatment was the feedstuffs provided. Ewes were fed a total mixed ration (ASe: $43 \%$ alfalfa hay, $19 \%$ dehydrated beet pulp, $18 \%$ whole corn, $8 \%$ barley straw, $6 \%$ soybean meal, and $6 \%$ condensed separator byproduct; HSe: $43 \%$ alfalfa hay, 25\% Se-enriched wheat mill run, $17 \%$ dehydrated beet pulp, $8 \%$ barley straw, and $6 \%$ condensed separator byproduct; both $2.45 \mathrm{kcal}$ metabolizable energy (ME)/kg and $10.4 \%$ metabolizable protein on a dry matter (DM) basis) during this period to achieve an ADG of 135 g/day (NRC, 1985). Pregnancy was determined 31 days after breeding via transrectal ultrasonography (Aloka, Wallingford, CT, USA). Eighty-four pregnant ewes (day 36 of gestation, $n=42$ per treatment) were then shipped from the USSES to the Animal Nutrition and Physiology Center at NDSU (1584 km; 14.5 h transit time) where they were housed for the remainder of the experiment.

At NDSU, ewes were individually housed in $0.91 \times 1.2 \mathrm{~m}$ pens with tenderfoot flooring (i.e. no bedding) in a temperature-controlled $\left(12-21^{\circ} \mathrm{C}\right)$, ventilated facility for the duration of the study. Lighting within the facility was automatically timed to mimic daylight patterns. Ewes remained on their Se treatments (actual intakes: ASe, $11.5 \mu \mathrm{g}$ Se/kg BW per day; HSe, $77.0 \mu \mathrm{g} \mathrm{Se} / \mathrm{kg}$ BW per day) and on day 40 of gestation were assigned randomly to one of three nutritional intake treatments supplying $60 \%$ (RES), $100 \%$ (CON), or $140 \%$ (HIGH) of NRC (1985) requirements for all nutrients except for Se.

Ewes had free access to water and a trace-mineralized salt block containing no additional Se (99\% maximum, 96\% minimum NaCl, 2000 mg/kg Mn, 1000 mg/kg Fe, 1000 mg/kg Mg, 500 mg/kg S, 250 mg/kg Cu, 100 mg/kg Co, 80 mg/kg Zn, 
and $70 \mathrm{mg} / \mathrm{kg} \mathrm{I;} \mathrm{Roto} \mathrm{Salt} \mathrm{Company,} \mathrm{Penn} \mathrm{Yan,} \mathrm{NY,} \mathrm{USA).}$ During gestation, diets were fed once daily at $0800 \mathrm{~h}$ in a complete pelleted ration $(0.48-\mathrm{cm}$ diameter), and three pellet formulations (ASe pellet, HSe pellet, and concentrated Se pellet; as previously described by Meyer et al. (2010)) were blended to meet Se and ME levels as dictated by Se and nutritional intake treatments for each ewe. As for diets fed during early gestation, the Se source of the HSe pellet was the previously described Se-enriched wheat mill run, and the Se source of the ASe pellet was feedstuffs used in the diet. ASe or HSe pellets were fed to ewes in the ASe and HSe treatments, respectively, to meet the $\mathrm{ME}$ level appropriate for their nutritional intake treatment. The HSe pellet was used in combination with the ASe pellet as needed to meet the targeted HSe and ME levels for each ewe. When the HSe pellet could not meet the HSe level for RES- or CON-fed ewes without exceeding the desired nutritional intake, a concentrated Se pellet with purified selenomethionine as its Se source was used to augment the Se supply. Nutrient requirements were based on NRC (1985) recommendations as described by Swanson et al. (2008), and diets were individually adjusted for BW and BW gain for each 14 day interval of gestation. Feed refusals were collected daily to calculate intake (feed offered minus feed refused), but ewes generally ate the feed offered daily and rarely left refusals. As ewes were housed indoors, and lambs would never be exposed to direct sunlight, all ewes were injected i.m. with vitamin A and D (2.0 ml/ewe; $500000 \mathrm{IU}$ vitamin A and 75000 IU vitamin $D_{3} / \mathrm{ml}$; Duravet, Blue Springs, MO, USA) on day 109 of gestation as a preventative measure for skeletal related issues.

\section{Parturition and lamb management}

Ewes were closely monitored during lambing, and lambs were removed immediately after parturition, before being allowed to suckle, so they could be reared on a common diet. Immediately after birth, lamb BW was measured (this was designated as initial BW or BW at birth), and an initial jugular blood sample was taken for hormone analysis. Lambs were then s.c. administered a vaccination against Clostridium perfringens types $C$ and $D$ and tetanus (Ultrabac CD; Pfizer Animal Health, New York, NY, USA), injected i.m. with vitamins $A$ and D (0.5 ml/lamb; $500000 \mathrm{IU}$ vitamin A and $75000 \mathrm{IU}$ vitamin $\mathrm{D}_{3} / \mathrm{ml}$; Duravet), and had their navels clipped and dipped in a 7\% iodine tincture.

Lambs were fed artificial colostrum (45\% crude protein; $15 \%$ crude fat; $0.15 \%$ crude fiber; $10 \%$ ash; $0.40 \%$ minimum, $0.90 \%$ maximum $\mathrm{Ca} ; 0.30 \%$ minimum, $0.50 \%$ maximum $\mathrm{NaCl} ; 1.0 \%$ minimum, $1.5 \%$ maximum $\mathrm{Na} ; 0.40 \% \mathrm{P}$; $0.75 \mathrm{mg} / \mathrm{kg}$ Se; $110000 \mathrm{IU} / \mathrm{kg}$ vitamin A; $2200 \mathrm{IU} / \mathrm{kg}$ vitamin $\mathrm{D}$; and $440 \mathrm{IU} / \mathrm{kg}$ vitamin E (DM basis); Acquire Colostrum Replacement; APC, Inc., Ankeny, IA, USA) within 30 min of birth and at six additional times by $20 \mathrm{~h}$ postpartum to achieve $10.64 \mathrm{~g} \mathrm{lgG} / \mathrm{kg} \mathrm{BW}(19.1 \mathrm{ml}$ colostrum $/ \mathrm{kg} \mathrm{BW}$ for the first two feedings and $25.5 \mathrm{ml}$ colostrum/kg BW in subsequent feedings; Meyer et al. 2010, Hammer et al. 2011). This dosage of IgG was calculated to provide $50 \mathrm{~g} \operatorname{lgG} / 4.7 \mathrm{~kg}$ BW based on the previous reports using a similar product (Quigley et al. 2002).

At $24 \mathrm{~h}$, a blood sample was collected via jugular venipuncture, and lambs were transitioned to milk replacer
(24\% crude protein; $30 \%$ crude fat; $0.10 \%$ crude fiber; $6.5 \%$ ash; $25 \%$ lactose; $0.50 \%$ minimum, $1.0 \%$ maximum Ca; $0.65 \% \mathrm{P} ; 2 \mathrm{mg} / \mathrm{kg}$ minimum, $6 \mathrm{mg} / \mathrm{kg}$ maximum $\mathrm{Cu} ; 0.3 \mathrm{mg} / \mathrm{kg}$ Se; $66000 \mathrm{IU} / \mathrm{kg}$ vitamin A; $22000 \mathrm{IU} / \mathrm{kg}$ vitamin $\mathrm{D}_{3}$; $330 \mathrm{IU} / \mathrm{kg}$ vitamin E (DM basis); Super Lamb Instant Milk Replacer; Merrick's, Inc., Middleton, WI, USA) fed from a bottle until a strong suckling response was observed (Meyer et al. 2010, Neville et al. 2010, Hammer et al. 2011). Once this occurred, the lambs were moved to a pen with free access to water and creep feed and adapted to a teat bucket system for ad libitum access to milk replacer (Meyer et al. 2010, Neville et al. 2010, Hammer et al. 2011). Tails were not docked, and male lambs were not castrated to prevent any decrease in performance caused by these procedures. Jugular blood samples were collected on days 3, 7, 14, and 19. On day 19, final weights and jugular blood samples were obtained from all lambs before necropsy. Percentage of BW change was calculated as follows: ((Final BW-Initial BW)/ Initial BW) $\times 100$, where initial BW was BW at birth.

\section{Necropsy}

On day $20.6 \pm 0.9$ (range: 19-21), lambs were killed by stunning. All internal organs, including the brain, liver, heart, lungs, total gastrointestinal complex, kidney, adrenal glands, gonads, uterus, thyroids, and spleen, were weighed using procedures similar to those described in Reed et al. (2007). Organ weight was divided by brain weight to assess asymmetric vs symmetric growth restriction, as the brain is spared during the time of maternal nutrient restriction used in this study (Anthony et al. 2003). For comparison, organ weight was also divided by EBW (live weight minus digesta weight), as our laboratory has previously reported (Reed et al. 2007, Swanson et al. 2008, Meyer et al. 2010, Neville et al. 2010).

\section{Cortisol analysis}

Lamb serum samples were analyzed for cortisol concentration as described previously (Lekatz et al. 2010). Briefly, serum samples $(10 \mu \mathrm{l})$ were assayed in duplicate by the chemiluminescence immunoassay (Immulite 1000; Siemens, Los Angeles, CA, USA). Within each assay, lesser, medium, and greater cortisol pools were assayed in duplicate (49.8 \pm 1.4 , $134.2 \pm 3.3$, and $343.5 \pm 5.2 \mathrm{ng} / \mathrm{ml}$, mean \pm s.E.M. for lesser, medium, and greater cortisol pools respectively). The sensitivity of the assay is $2 \mathrm{ng} / \mathrm{ml}$. The intra- and interassay coefficients of variation (CV) were 6.2 and $6.4 \%$ respectively.

\section{Thyroid hormone analysis}

$\mathrm{T}_{4}$ and $\mathrm{T}_{3}$ concentrations were determined by the chemiluminescence immunoassay using the Immulite 1000 (Siemens) as we have described ( $\mathrm{O}^{\prime} \mathrm{Neil}$ et al. 2009). Within each assay, $\mathrm{T}_{4}$ and $\mathrm{T}_{3}$ pools were assayed in duplicate $(24.5 \pm 0.1,78.8$ \pm 0.1 , and $105.9 \pm 0.2 \mathrm{ng} / \mathrm{ml}$ and $0.88 \pm 0.07,1.53 \pm 0.05$, and $2.98 \pm 0.05 \mathrm{ng} / \mathrm{ml}$, mean \pm s.E.M. for lesser, medium, and greater pools for $T_{4}$ and $T_{3}$ respectively). Fifteen microliter and $25 \mu \mathrm{l}$ serum samples were assayed in duplicate for $\mathrm{T}_{4}$ and $T_{3}$ respectively. The sensitivity of the $T_{4}$ and $T_{3}$ assays is 4.0 
and $0.35 \mathrm{ng} / \mathrm{ml}$ respectively. The intra-assay CV was 3.4 and $3.7 \%$ for $\mathrm{T}_{4}$ and $\mathrm{T}_{3}$, respectively, and the interassay $\mathrm{CV}$ was 7.8 and $13.4 \%$ for $T_{4}$ and $T_{3}$ respectively.

\section{Statistical analysis}

Twins ( $n=3$ sets) were removed from the data set (one set each for HSe-RES, ASe-HIGH, and HSe-HIGH). One ewe (ASe-RES) was not pregnant and removed from the study. Five lambs died between birth and necropsy, and therefore, only BW at birth was included in the data set for these animals. Thus, the final lamb numbers used for each treatment were as follows: ASeRES (13; six females and seven males), ASe-CON (14; ten females and four males), ASe-HIGH (13; seven females and six males), HSe-RES (13 at birth; eight females and five males; ten at necropsy; six females and four males), HSe-CON (14 at birth; 11 females and three males; 13 at necropsy; ten females and three males), and HSe-HIGH (13; eight females and five males).

Data were normally distributed and analyzed using the MIXED procedure of SAS version 9.1 (SAS Institute, Inc., Cary, NC, USA). Ewe gestational dietary Se supplementation (ASe vs HSe), nutritional intake (RES vs CON vs $\mathrm{HIGH}$ ), and all interactions were used as fixed effects in the model. As maternal nutritional treatments can influence phenotypes of males and females differently, offspring sex was included in the model as a random effect, and interactions with sex were tested. When appropriate (i.e. for percentage weight change and hormone data), day of age was also included in the model. For the variables' percentage weight change and $\mathrm{T}_{4}$ (from days 3 to 19), the first-order autoregressive covariance structure was used. For the variables' cortisol and $T_{3}$ (from days 3 to 19), the unstructured covariance structure was used. Interactions were removed from the model when $P>0.15$ but were retained in the model if they were of interest. Means were separated using least significant differences and considered significant when $P \leq 0.05$ or tendencies when $0.05<P<0.10$. In the absence of interactions $(P>0.10)$, main effects $(P<0.10)$ are reported; otherwise interactive means are discussed.

\section{Declaration of interest}

The authors declare that there is no conflict of interest that could be perceived as prejudicing the impartiality of the research reported.

\section{Funding}

This project was partially supported by National Research Initiative Competitive Grant no. 2005-35206-15281 from the USDA Cooperative State Research, Education, and Extension Service to J S Caton, D A Redmer, and K A Vonnahme and by a LEAP grant to $\mathrm{K}$ A Vonnahme from National Science Foundation Grant \#HRD-0811239 to the NDSU Advance FORWARD program. We would like to thank members of the Reproductive Physiology and Ruminant Nutrition laboratories for their assistance with animal care and data collection.

\section{References}

Anthony RV, Scheaffer AN, Wright CD \& Regnault TR 2003 Ruminant models of prenatal growth restriction. Reproduction 61 183-194.

Arthur JR, Nicol F \& Beckett GJ 1990 Hepatic iodothyronine 5'-deiodinase. The role of selenium. Biochemical Journal 272 537-540.

Bauer R, Walter B, Hoppe A, Gaser E, Lampe V, Kauf E \& Zweiner U 1998 Body weight distribution and organ size in newborn swine (Sus scrofa domestica) - a study describing an animal model for asymmetrical intrauterine growth retardation. Experimental and Toxicologic Pathology 50 59-65. (doi:10.1016/S0940-2993(98)80071-7)

Beckett GJ, Beddows SE, Nicol PC \& Arthur JR 1987 Inhibition of hepatic deiodination of thyroxine is caused by selenium deficiency in rats. Biochemical Journal 248 443-447.

Challis JRG, Matthews SG, Gibb W \& Lye SJ 2000 Endocrine and paracrine regulation of birth at term and preterm. Endocrine Reviews 21 514-550. (doi:10.1210/er.21.5.514)

Contempre B, Jauniaux E, Calvo R, Jurkovic D, Campbell S \& Morreale de Esocbar G 1993 Detection of thyroid hormone in human embryonic cavities during the first trimester of gestation. Journal of Clinical Endocrinology and Metabolism 77 1719-1722. (doi:10.1210/jc.77.6. 1719)

DeBlasio MJ, Gatford KL, Robinson JS \& Owens JA 2006 Placental restriction alters circulating thyroid hormone in young lamb postnatally. American Journal of Physiology. Regulatory, Integrative and Comparative Physiology 291 R1016-R1024. (doi:10.1152/ajpregu.00103.2006)

Fowden AL \& Forhead AJ 2004 Endocrine mechanisms of intrauterine programming. Reproduction 127 515-526. (doi:10.1530/rep.1.00033)

Fry S, Meyer AM, Reed JJ, Neville TL, Taylor JB, Reynolds LP, Redmer DA, Vonnahme KA \& Caton JS 2010 Effects of maternal nutritional plane and selenium supply on jejuna crypt cell proliferation and villous morphology in offspring at 20 days of age. Journal of Animal Science 88 (ESuppl 3) 143.

Hammer CJ, Thorson JF, Vonnahme KA, Meyer AM, Redmer DA, Luther JS, Neville TL, Reed JJ, Reynolds LP \& Caton JS 2011 Effects of maternal selenium supply and plane of nutrition during gestation on passive transfer of immunity and health in neonatal lambs. Journal of Animal Science 89 3690-3698. (doi:10.2527/jas.2010-3724)

Jensen EC, Harding JE, Bauer MK \& Gluckman PD 1999 Metabolic effects of IGF-I in the growth retarded fetal sheep. Journal of Endocrinology 161 485-494. (doi:10.1677/joe.0.1610485)

Johnston NE \& Stewart JA 1986 The effect of glucocorticoids and prematurity on absorption of colostral immunoglobulins in the calf. Australian Veterinary Journal 63 191-192. (doi:10.1111/j.1751-0813. 1986.tb02973.x)

Kamada H, Nonaka I, Ueda Y \& Murai M 2007 Selenium addition to colostrum increases immunoglobulin $\mathrm{G}$ absorption by newborn calves. Journal of Dairy Science 90 5665-5670. (doi:10.3168/jds.2007-0348)

Knight DA \& Tyznik WJ 1990 The effect of dietary selenium on humoral immunocompetence of ponies. Journal of Animal Science $\mathbf{6 8}$ 1311-1317.

Lang U, Baker RS, Braems G, Zygmunt M, Künzel W \& Clark KE 2003 Uterine blood flow - a determinant of fetal growth. European Journal of Obstetrics, Gynecology, and Reproductive Biology 110 S55-S61. (doi:10.1016/S0301-2115(03)00173-8)

Lekatz LA, Caton JS, Taylor JB, Reynolds LP, Redmer DA \& Vonnahme KA 2010 Maternal selenium supplementation and timing of nutrient restriction in pregnant sheep: effects on maternal endocrine status and placental characteristics. Journal of Animal Science 88 955-971. (doi:10.2527/jas.2009-2152)

Lekatz LA, Wu G, Caton JS, Taylor JB, Reynolds LP, Redmer DA \& Vonnahme KA 2011 Maternal selenium supplementation and timing of nutrient restriction in pregnant sheep: impacts on nutrient availability to the fetus. Journal of Animal Science 89 59-76. (doi:10.2527/jas.20103015)

Mellor DJ \& Pearson RA 1977 Some changes in the composition of blood during the first 24 hours after birth in normal and growth retarded lambs. Annales de Recherches Vétérinaires. Annals of Veterinary Research $\mathbf{8 4}$ 460-467.

Meyer AM, Reed JJ, Neville TL, Taylor JB, Hammer CJ, Reynolds LP, Redmer DA, Vonnahme KA \& Caton JS 2010 Effects of plane nutrition 
and selenium supply on ewe and neonatal offspring performance, body composition, and serum selenium. Journal of Animal Science $\mathbf{8 8}$ 1786-1800. (doi:10.2527/jas.2009-2435)

Meyer AM, Reed JJ, Neville TL, Thorson JF, Maddock-Carlin KR, Taylor JB, Reynolds LP, Redmer DA, Luther JS, Hammer CJ et al. 2011 Nutritional plane and selenium supply during gestation affect yield and nutrient composition of colostrum and milk in primiparous ewes. Journal of Animal Science 89 1627-1639. (doi:10.2527/jas.2010-3394)

Morrison JL 2008 Sheep models of intrauterine growth restriction: fetal adaptations and consequences. Clinical and Experimental Pharmacology \& Physiology 35 730-743. (doi:10.1111/j.1440-1681.2008. 04975.x)

Neville TL, Caton JS, Hammer CJ, Reed JJ, Luther JS, Taylor JB, Redmer DA, Reynolds LP \& Vonnahme KA 2010 Ovine offspring growth and diet digestibility are influenced by maternal selenium supplementation and nutritional intake during pregnancy despite a common postnatal diet. Journal of Animal Science 88 3645-3656. (doi:10.2527/jas.2009-2666)

Newton ER, Kennedy JL Jr, Louis F, Cetrulo CL, Sbarra A \& Feingold M 1987 Obstetric diagnosis and perinatal mortality. American Journal of Perinatology 4 300-304. (doi:10.1055/s-2007-999795)

O'Neil MR, Lardy GP, Wilson ME, Lemley CO, Reynolds LP, Caton JS \& Vonnahme KA 2009 Estradiol-17beta and linseed meal interact to alter visceral organ mass and hormone concentrations from ovariectomized ewes. Domestic Animal Endocrinology 37 148-158. (doi:10.1016/ j.domaniend.2009.05.001)

Pearce JM \& Campbell S 1985 Intrauterine growth retardation. Birth Defects Original Article Series 21 109-130.

Poppe K \& Ginoer D 2003 Thyroid autoimmunity and hypothyroidism before and during pregnancy. Human Reproduction Update 9 149-161. (doi:10.1093/humupd/dmg012)

Quigley JD, Carson AF \& Polo J 2002 Immunoglobulin derived from bovine plasma as a replacement for colostrum in newborn lambs. Veterinary Therapeutics: Research in Applied Veterinary Medicine 3 262-269.

Reed JJ, Ward MA, Vonnahme KA, Neville TL, Julius SL, Taylor JB, Redmer DA, Grazul-Bilska AT, Reynolds LP \& Caton JS 2007 Effects of selenium supply and dietary restriction on maternal and fetal body weight, visceral organ mass, cellularity estimates, and jejunal vascularity in pregnant ewe lambs. Journal of Animal Science 85 2721-2733. (doi:10.2527/jas.2006-785)

Reynolds LP, Borowicz PP, Caton JS, Vonnahme KA, Luther JS, Hammer CJ, MaddockCarlin KR, Grazul-Bilska AT \& Redmer DA 2010 Developmental programming: the concept, large animal models, and the key role of utero-placental vascular development. Invited review. Journal of Animal Science 88 (Suppl 13) E61-E72. (doi:10.2527/jas.2009-2359)
Saintonge J \& Rosso P 1981 Placental blood flow and transfer of nutrient analogs in large, average, and small guinea pig littermates. Pediatric Research 15 152-156. (doi:10.1203/00006450-198102000-00014)

Sangild PT, Jacobsen H, Schmidt M, Fowden AL, Avery B \& Greve T 1999 IgG and cortisol levels in newborn premature IVP calves. Theriogenology 511 331. (doi:10.1016/S0093-691X(99)91890-3)

Schneider F, Kanitz E, Gerrad ED, Kuhn G, Brussow KP, Numberg K, Fiedler I, Numberg G, Ender K \& Rehfeldt C 2002 Administration of recombinant porcine somatotropin (rpST) changes hormone and metabolic status during early pregnancy. Domestic Animal Endocrinology 23 455-474. (doi:10.1016/S0739-7240(02)00175-3)

Stott GH 1980 Immunoglobulin absorption in calf neonates with special considerations of stress. Journal of Dairy Science 63 681-688. (doi:10. 3168/jds.S0022-0302(80)82990-0)

Swanson TJ, Hammer CJ, Luther JS, Carlson DB, Taylor JB, Redmer DA, Neville TL, Reed JJ, Reynolds LP, Caton JS et al. 2008 Effects of gestational plane of nutrition and selenium supplementation on mammary development and colostrum quality in pregnant ewe lambs. Journal of Animal Science 86 2415-2423. (doi:10.2527/jas.2008-0996)

Vonnahme KA, Luther JS, Reynolds LP, Hammer CJ, Carlson DB, Redmer DA \& Caton JS 2010 Impacts of maternal selenium and nutritional level on growth, adiposity, and glucose tolerance in female offspring in sheep. Domestic Animal Endocrinology 39 240-248. (doi:10.1016/j.domaniend.2010.06.005)

Wallace JM, Bourke DA, Aitken RP, Palmer RM, Da Silva P \& Cruickshank MA 2000 Relationship between nutritionally-mediated placental growth restriction and fetal growth, body composition and endocrine status during late gestation in adolescent sheep. Placenta $\mathbf{2 1}$ 100-108. (doi:10.1053/plac.1999.0440)

Widdowson EM 1971 Intrauterine growth retardation in the pig. Part I. Organ size and cellular development at birth and after growth to maturity. Biology of the Neonate 19 329-340. (doi:10.1159/000240427)

Wu G, Bazer FW, Wallace JM \& Spencer TE 2006 Intrauterine growth retardation: implications for the animal sciences. Journal of Animal Science 84 2316-2337. (doi:10.2527/jas.2006-156)

Received 16 August 2011

First decision 16 September 2011

Revised manuscript received 28 March 2012

Accepted 18 April 2012 